\title{
Pulmonary vascular density: comparison of findings on computed tomography imaging with histology
}

\author{
Farbod N. Rahaghi, ${ }^{1,6}$, Gemma Argemín ${ }^{2,6}$, Pietro Nardelli ${ }^{3}$, \\ David Domínguez-Fandos ${ }^{2}$, Pedro Arguis ${ }^{4}$, Víctor I. Peinado ${ }^{2,5}$, James C. Ross ${ }^{3}$, \\ Samuel Y. Ash ${ }^{1}$, Isaac de La Bruere ${ }^{1}$, Carolyn E. Come ${ }^{1}$, Alejandro A. Diaz ${ }^{1}$, \\ Marcelo Sánchez ${ }^{4}$, George R. Washko ${ }^{1,7}$, Joan Albert Barberà ${ }^{2,5,7}$ and \\ Raúl San José Estépar (1) ${ }^{1,7}$
}

Affiliations: ${ }^{1}$ Pulmonary and Critical Care Division, Dept of Medicine, Brigham and Women's Hospital, Boston, MA, USA. ${ }^{2}$ Dept of Pulmonary Medicine, Hospital Clínic-IDIBAPS, University of Barcelona, Barcelona, Spain. ${ }^{3}$ Dept of Radiology, Harvard School of Medicine, Boston, MA, USA. ${ }^{4}$ Dept of Radiology, Hospital ClínicIDIBAPS, University of Barcelona, Barcelona, Spain. ${ }^{5}$ Biomedical Research Networking Center for Respiratory Diseases (CIBERES), Madrid Spain. ${ }^{6}$ Equal contributors on this article. ${ }^{7}$ Equal contributors on this article.

Correspondence: Farbod N. Rahaghi, Pulmonary and Critical Care Division, Dept of Medicine, Brigham and Women's Hospital, 75 Francis Street, Boston, MA 02115, USA. E-mail: frahaghiabwh.harvard.edu

@ERSpublications

Vascular density on CT imaging correlates with vascular density in histology in smokers. Imagingbased quantification of pulmonary vasculature provides a noninvasive method to study the multiscale effects of smoking on the pulmonary circulation. http://bit.ly/2WprQe8

Cite this article as: Rahaghi FN, Argemí G, Nardelli P, et al. Pulmonary vascular density: comparison of findings on computed tomography imaging with histology. Eur Respir J 2019; 54: 1900370 [https://doi.org/ 10.1183/13993003.00370-2019].

\section{ABSTRACT}

Background: Exposure to cigarette smoke has been shown to lead to vascular remodelling. Computed tomography (CT) imaging measures of vascular pruning have been associated with pulmonary vascular disease, an important morbidity associated with smoking. In this study we compare CT-based measures of distal vessel loss to histological vascular and parenchymal changes.

Methods: A retrospective review of 80 patients who had undergone lung resection identified patients with imaging appropriate for three-dimensional (3D) vascular reconstruction $(n=18)$ and a second group for two-dimensional (2D) analysis $(n=19)$. Measurements of the volume of the small vessels (3D) and the cross-sectional area of the small vessels $\left(<5 \mathrm{~mm}^{2}\right.$ cross-section) were computed. Histological measures of cross-sectional area of the vasculature and loss of alveoli septa were obtained for all subjects.

Results: The 2D cross-sectional area of the vasculature on CT imaging was associated with the histological vascular cross-sectional area $(\mathrm{r}=0.69 ; \mathrm{p}=0.001)$. The arterial small vessel volume assessed by CT correlated with the histological vascular cross-sectional area $(\mathrm{r}=0.50 ; \mathrm{p}=0.04)$, a relationship that persisted even when adjusted for CT-derived measures of emphysema in a regression model.

Conclusions: Loss of small vessel volume in CT imaging of smokers is associated with histological loss of vascular cross-sectional area. Imaging-based quantification of pulmonary vasculature provides a noninvasive method to study the multiscale effects of smoking on the pulmonary circulation. 


\section{Introduction}

Chronic exposure to cigarette smoke can result in emphysematous destruction of the lung parenchyma, inflammation, destruction of the airways and remodelling of the pulmonary vasculature $[1,2]$. While some smokers suffering from the latter process may develop overt pulmonary hypertension [3-6], pulmonary vascular remodelling in smokers may be an integral part of the pathway from lung health to advanced disease [7-10]. As such, histological and imaging-based metrics of this process may provide useful features for detecting susceptibility to disease and stratifying its severity.

Remodelling of the pulmonary vasculature has been observed on histological and in vivo image-based investigations $[2,7,11-16]$. These studies have demonstrated that the loss of microvasculature is associated with the loss of alveolar septa, characteristic of emphysema [11, 17]. In clinical investigations, quantitative measures of small vessel cross-sectional area have been shown to be related to multiple parameters of smoking-related pulmonary vascular disease severity $[11,12,18,19]$. There is little data, however, linking histopathological data to those obtained from computed tomography (CT) scans.

In this study we sought to determine the relationship between CT-based measures of small vessel volume and the loss of microvasculature on histological examination. We hypothesised that small vessel loss on CT was related to microvascular pruning. Establishing such a link would further substantiate CT measures of the pulmonary vasculature as providing insight into lung pathology in smokers.

\section{Methods}

\section{Subject selection}

Subjects from Hospital Clínic (Barcelona, Spain) were selected by review of CT scans in a prospective cohort of 80 subjects who had undergone surgical lung resection for lung cancer, for which histological samples had been acquired and processed as part of a study of histology in smokers. The study was approved by the ethics committee of Hospital Clínic (RE 2012/7982) and all subjects provided written informed consent to participate in the study.

Two cohorts were selected with no overlap between the two cohorts. The first cohort had CT scans with thin sections sufficient for three-dimensional (3D) reconstruction (1.0-1.5 mm thick) obtained using the same scanner (Emotion 16; Siemens, Erlangen, Germany) with similar reconstruction kernels (B30s, B31s and B41s). This constituted the 3D analysis cohort. The second cohort consisted of subjects having CT scans with similar reconstruction kernels (B70, B75 and B80) and thickness between 1 and $2 \mathrm{~mm}$ (mostly $2 \mathrm{~mm}$ ), but to which a two-dimensional (2D) measure of small vessel density would be then applied. Criteria for exclusion included poor quality due to motion artefacts, missing part of the scan or significant infiltrates in the resected lung that would confound analysis. Characteristics of the subjects in the two cohorts are shown in table 1.

\begin{tabular}{lccc}
\multicolumn{4}{l}{ TABLE 1 Demographics and clinical characteristics of the two cohorts } \\
& All subjects & Cohort for 3D reconstruction & Cohort for 2D analysis \\
\hline Subjects & 37 & 18 & 19 \\
Female/male & $21 / 16$ & $12 / 6$ & $9 / 10$ \\
Age years & $66(12)$ & $68(14)$ & $64(15)$ \\
BMI kg.m & $27(6)$ & $26(4)$ & $28(5)$ \\
Smoker & 30 & 16 & 14 \\
Smoking exposure pack-years & $40(45)$ & $40(37)$ & $45(56)$ \\
FEV1/FVC \% & $68(12)$ & $65(7)$ & $73(11)$ \\
FEV $\%$ pred & $77(20)$ & $71(18)$ & $85(19)$ \\
FVC \% pred & $85(20)$ & $85(23)$ & $84(16)$ \\
TLC \% pred & $94(17)$ & $88(22)$ & $95(12)$ \\
DLco \% pred & $74(18)$ & $65(13)$ & $77(16)$ \\
PaO $\mathbf{m m H g}$ & $83(12)$ & $83(6)$ & $78(14)$ \\
COPD & $21(57 \%)$ & $13(72 \%)$ & $8(42 \%)$
\end{tabular}

Data are presented as $\mathrm{n}$ or median (interquartile range), unless otherwise stated. 3D: three-dimensional; 2D: two-dimensional; BMI: body mass index; FEV1: forced expiratory volume in $1 \mathrm{~s}$; FVC: forced vital capacity; TLC: total lung capacity; $D$ LCO: diffusing capacity of the lung for carbon monoxide; $P_{\mathrm{aO}}$ : arterial oxygen tension; COPD: chronic obstructive pulmonary disease. 


\section{Histological processing}

Lung tissue samples were collected prospectively and processed after surgery. Part of the histological samples had been stored at the Hospital Clínic Core of the Pulmonary Biobank Consortium of the Biomedical Research Networking Center for Respiratory Diseases (CIBERES). Briefly, immediately after resection, fresh lung tissue slices 3-4 mm thick were washed 3 times (10 min each) in cold PBS in order to distend collapsed areas of parenchyma and clean off embedded blood. Paraffin-embedded lung tissue blocks were sectioned into $5 \mu \mathrm{m}$ thick slices that were stained with haematoxylin and eosin. Slices were imaged at $\times 40$ magnification using a bright field microscope (model DM 5000) connected to a Leica camera (model DFC 500) (Leica Microsystems, Wetzlar, Germany). Four images per subject were acquired and analysed with Image-Pro software (www.mediacy.com). The cross-sectional area of vessels $<500 \mu \mathrm{m}$ diameter was calculated manually and expressed as a percentage of the total area of the four lung tissue images (\%hist-VCSA) (figure $1 \mathrm{a}$ and b). Vessels with a diameter $\geqslant 500 \mu \mathrm{m}$ or with a longitudinal disposition (ratio major/minor diameter $>2: 1$ ) were discarded. The proportions of lung tissue structures (alveolar septa, vessels and bronchioles) were estimated by using a point counting method. To this end, a grid with 108 intersection points was superimposed on the microscopic field at $\times 40$ magnification and each intersection point was assigned to alveolar septum, vessel, bronchiole or airspace. Counts superimposed on each structure were then divided by the total number of points on the grid and expressed as a percentage (figure 1c). 10 pulmonary muscular arteries per subject were identified, and the external and internal elastic laminas and the inner aspect of the intima were outlined. The areas occupied by the muscular layer, the intimal layer and the lumen were computed and expressed as a percentage of the measured total area. Visual examples of these processes are shown in figure $1 \mathrm{~d}-\mathrm{f}$.

\section{CT vascular analysis \\ $2 D$ analysis}

The cross-sectional area of the vasculature was computed as described previously using ImageJ version 1.46r [20]. A visual example of this process is shown in supplementary figure S1. In summary, circle-like objects with an area $<5 \mathrm{~mm}^{2}$ were identified on an individual slice; the aggregate area of these objects was then divided by the lung cross-sectional area in the same slice yielding the CSA5\%. 2D analysis was
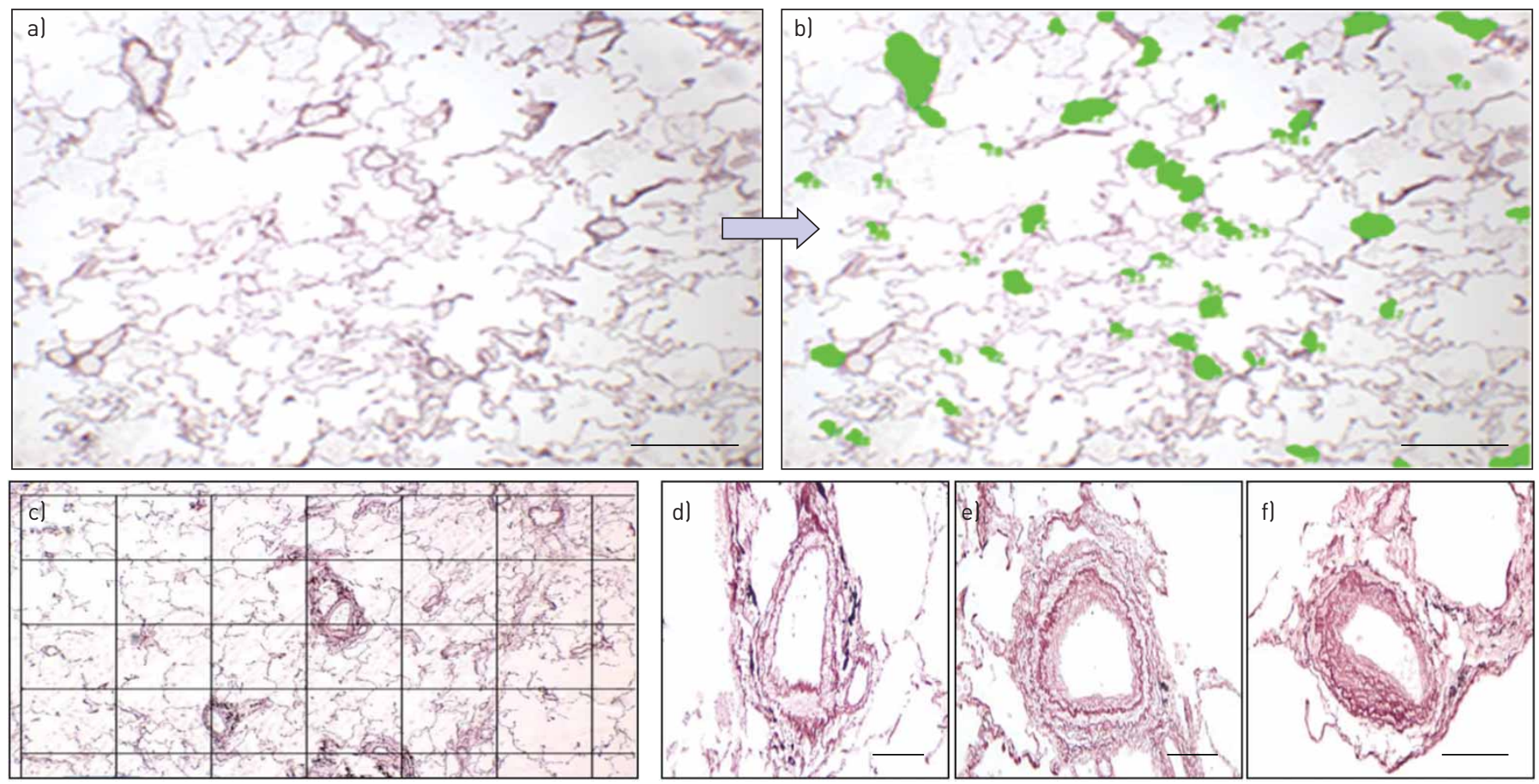

FIGURE 1 a) Micrograph of a section prepared from excised lung tissue of a patient undergoing lung resection for a tumour. b) Vessels were identified manually and coloured in. The cross-sectional area of vessels $<500 \mu \mathrm{m}$ was summed and divided by the entire field of view to derive the histological vessel cross-sectional area (\%hist-VCSA). Scale bar: $500 \mu \mathrm{m}$. c) To measure the proportion of tissue structures, a grid was superimposed on the microscopic field and intersection points corresponding to alveolar septa, vessels and bronchioles were identified. Grid cell size: $100 \mu \mathrm{m} \times 150 \mu \mathrm{m} . \mathrm{d}-\mathrm{f}$ ) The thickness of the wall, wall components and lumen was measured from individual arteries: example arteries from d) a nonsmoker, e) a subject with Global Initiative for Chronic Obstructive Lung Disease (GOLD) stage II chronic obstructive pulmonary disease (COPD) and f) a subject with GOLD stage III COPD. Scale bar: $100 \mu \mathrm{m}$. 
performed on the same slide as the histological sample. For patients with upper lobe resections or pneumonectomies, a section at the same level as the aortic arch was used. For those with lower lobe resections, a CT slice at the level of the carina was used. Images were not blurred prior to analysis.

\section{$3 D$ analysis}

The lungs were automatically segmented from the chest wall and $3 \mathrm{D}$ reconstructions of the vasculature were created for the entirety of the lung [21-23]. The vessel size estimation was performed with a deep learning-based approach that used generative models of the vessel, estimating the radius from images in the orthogonal plane of the vessel [24]. The volume of vasculature was computed as a function of the cross-sectional area of the vessels, and the total volume in small vessels was derived and termed BV5; a cut-off of $5 \mathrm{~mm}^{2}$ was used consistent with prior investigations. BV5 was divided by the volume of the lung to arrive at a vascular density (BV5/LV). As hyperinflation of the lung can confound the use of lung volume in smokers, a nonemphysematous lung volume (NELV) was also computed using the following equation: NELV=lung volume-total vessel volume-volume of emphysema.

The volume of emphysematous tissue was computed using the percentage of low-attenuation area less than $-950 \mathrm{HU}$ (\%LAA-950), as described previously [25, 26]. This was then used to arrive at a second normalised measure of small vessel volume, BV5/NELV.

Automated arterial/venous segmentation was performed using a convolutional neural network algorithm described previously [27]. In order to validate the method in this cohort, six cases were randomly selected and reviewed by tracing the path of the vessels from their origins to the pulmonary artery and the left atrium. Disagreements between the automated and manual method were measured segment by segment. The blood vessel volumes were then computed separately for the arterial and venous vasculature.

\section{Statistical analysis}

Data are presented as means and standard deviations. All statistical analyses were performed using $\mathrm{R}$ version 3.5 (www.r-project.org). Correlations were evaluated using Pearson and Spearman correlations where appropriate. p-values $<0.05$ were considered statistically significant.

\section{Results}

From a total of 80 subjects with appropriate histological samples, 18 subjects were identified with adequate imaging for $3 \mathrm{D}$ reconstruction and 19 subjects were identified for $2 \mathrm{D}$ vascular analysis. The demographics, smoking history and pulmonary function test results for these subjects are shown in table 1 and supplementary table S1. Table 2 and supplementary table 2 show the results of the histological assessments.

Volumetric reconstructions of the pulmonary vasculature were performed in each subject. Examples of two different 3D reconstructions with low and high BV5/LV are shown in figure 2. The CT-derived vascular ratios (BV5/LV and BV5/NELV) that describe vascular density changes are summarised in table 3.

\begin{tabular}{lccc} 
TABLE 2 Histological measures of the two cohorts & & \\
& $\begin{array}{c}\text { All } \\
\text { subjects }\end{array}$ & $\begin{array}{c}\text { Cohort for 3D } \\
\text { reconstruction }\end{array}$ & $\begin{array}{c}\text { Cohort for 2D } \\
\text { analysis }\end{array}$ \\
\hline Subjects & 37 & 18 & 19 \\
Estimated proportion of lung structures & & & \\
$\quad$ Alveolar space \% & $81(8.8)$ & $83(6.1)$ & $80(11)$ \\
$\quad$ Alveolar septa \% & $14(6.8)$ & $14(5.6)$ & $14(6.1)$ \\
Vessels \% & $4.2(2.8)$ & $4.1(1.4)$ & $4.4(4.2)$ \\
$\quad$ Bronchi \% & $0.5(0.7)$ & $0.5(0.7)$ & $0.0(0.7)$ \\
Cross-sectional area of vessels <500 $\mu \mathrm{mm}$ & $6.3(2.2)$ & $6.3(1.7)$ & $6.1(3.1)$ \\
diameter \% total area & & & \\
Morphometry of pulmonary muscular & & & $278(97.5)$ \\
arteries & $277(96.0)$ & $266(93.7)$ & $41(18)$ \\
External diameter $\mu$ m & $41(16)$ & $41(9)$ & $28(10)$ \\
Lumen \% vessel area & $30(11)$ & $31(12)$ & $23(9)$ \\
Intima \% vessel area & $24(7)$ & $25(6)$ & \\
Muscularis \% vessel area & & & \\
\hline
\end{tabular}

Data are presented as $\mathrm{n}$ or median (interquartile range). 3D: three-dimensional; 2D: two-dimensional. 


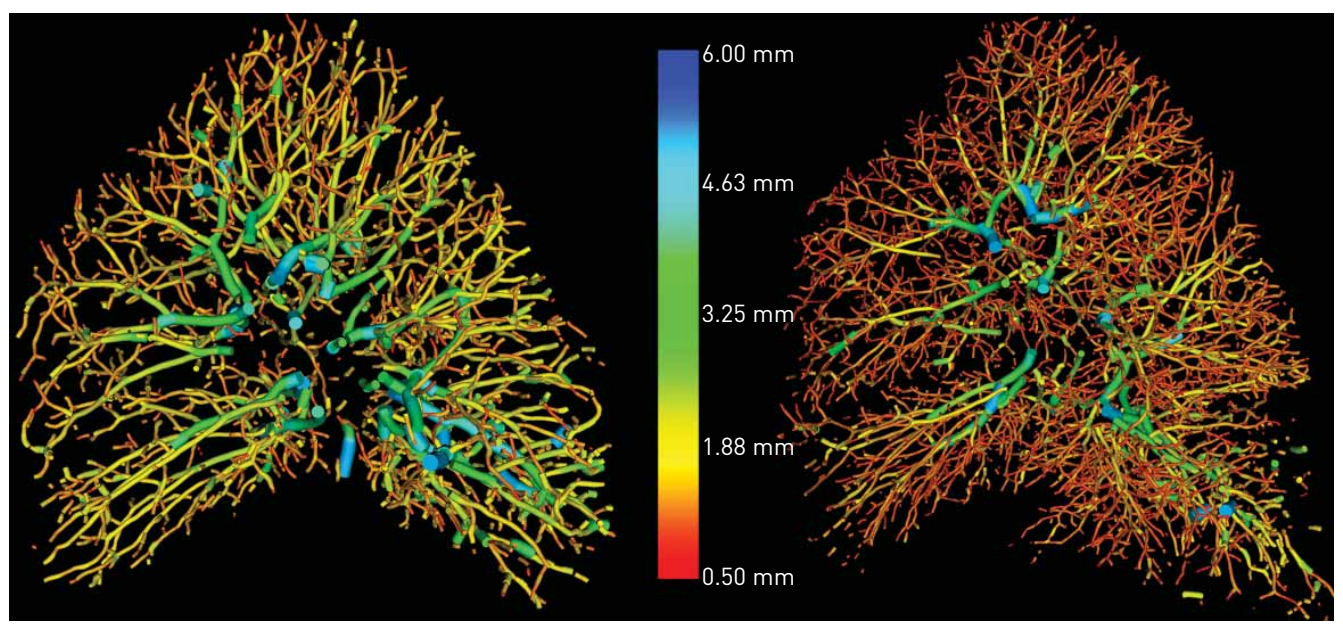

FIGURE 2 Examples of three-dimensional vascular reconstructions in two subjects both with Global Initiative for Chronic Obstructive Lung Disease stage II chronic obstructive pulmonary disease. The colour indicates the radius of the vessel, with red colouring representing smaller vessels. Imaging of the subject on the left shows proximal dilation and loss of distal vasculature compared with the subject on the right. On histology, the subject on the left had a lower histological vessel cross-sectional area (\%hist-VCSA 6.3 versus 8.6).

The relationships between imaging and histological metrics of septal and vascular densities in the $2 \mathrm{D}$ cohort are shown in figure 3. There was a significant positive correlation $(\mathrm{r}=0.69 ; \mathrm{p}=0.001)$ between \%hist-VCSA and CSA5\%. In addition, CSA5\% was positively correlated with the estimated proportion of alveolar septa $(\mathrm{r}=0.57 ; \mathrm{p}=0.01)$.

Arterial/venous segmentation was performed successfully for all 3D cases and comparison with manual segmentation in six randomly selected cases yielded an agreement of 96.7\%. An example of an arterial/ venous segmentation is shown in figure 4 . Given a number of outlying points, nonparametric (Spearman) correlation was used to evaluate the relationship between arterial small vessel volume (arterial BV5/LV) and \%hist-VCSA. There was a statistically significant relationship between arterial BV5/LV and \%hist-VCSA ( $\mathrm{r}=0.50 ; \mathrm{p}=0.04)$ which was not observed in the venous counterpart (venous BV5/LV) $(\mathrm{r}=0.08 ; \mathrm{p}=0.74)$ (figure $5 \mathrm{a}$ and $\mathrm{c})$. Additionally, there was a relationship between arterial/venous small vessel volume and percentage of alveolar septa, although this only reached statistical significance for the

$\begin{array}{lc}\text { TABLE } 3 \text { Computed tomography-based imaging measures } & \\ \text { Measures in the 2D cohort } \mathbf{( n = 1 9 )} & 2.44(1.16) \\ \text { 2D CSA5\% } & \\ \text { Measures in the 3D cohort } \mathbf{( n = 1 8 )} & 0.3(1.4) \\ \text { \%LAA-950 } & 4.5(1.4) \\ \text { LV L } & 889(212) \\ \text { Lung mass g } & 4.25(1.24) \\ \text { NELV L } & \\ \text { Measures of vascular volume } & 34.9(16.1) \\ \text { BV5 mL } & 78.5(28.1) \\ \text { TBV mL } & 7.9(0.85) \\ \text { BV5/LV } & 16.9(2.7) \\ \text { TBV/LV } & 8.3(13.7) \\ \text { BV5/NELV } & 18.1(3.0) \\ \text { TBV/NELV } & 19.9(8.6) \\ \text { Arterial BV5 mL } & 4.4(0.8) \\ \text { Arterial BV5/LV } & 4.7(0.9) \\ \text { Arterial BV5/NELV }\end{array}$

Data are presented as median (interquartile range). 2D: two-dimensional; CSA5\%: aggregate area of circle-like objects with an area $<5 \mathrm{~mm}^{2}$ divided by the lung cross-sectional area in the same slice; 3D: three-dimensional; \%LAA-950: percentage low-attenuation area less than $-950 \mathrm{HU}$; LV: lung volume; NELV: nonemphysematous lung volume; BV5: blood vessel volume in vessels with cross-sectional area $<5 \mathrm{~mm}^{2}$; TBV: total blood vessel volume. 

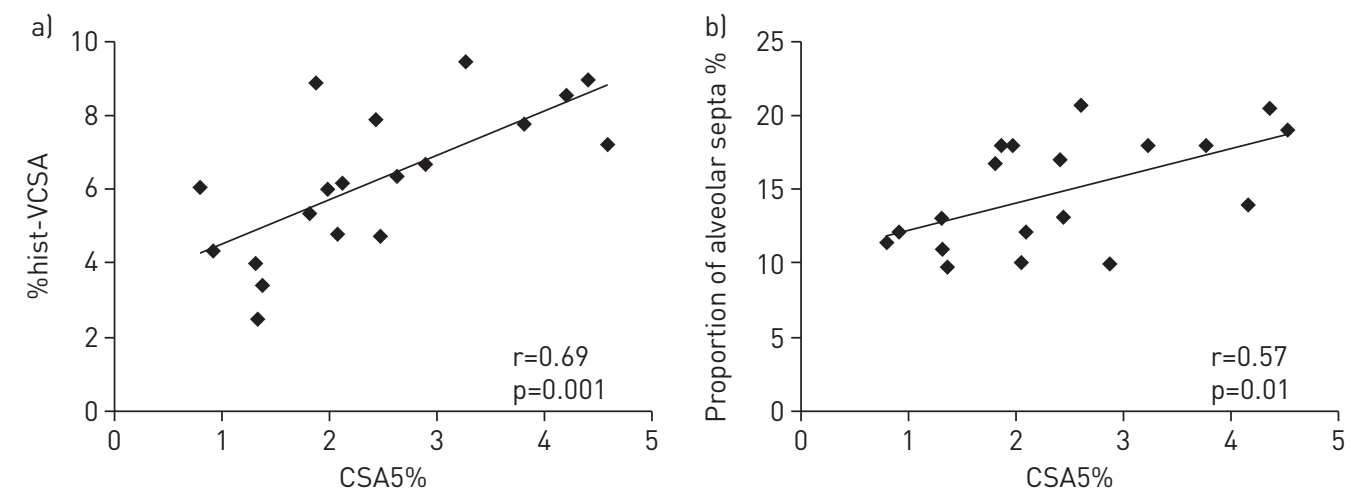

FIGURE 3 Summary of results from the two-dimensional analysis showing a correlation between cross-sectional area of vessels of $<5 \mathrm{~mm}^{2}$ (CSA5\%) assessed by computed tomography scan and a) histological metrics of small vessel (diameter $<500 \mu \mathrm{m}$ ) density (histological vessel cross-sectional area (\%hist-VCSA)] and b) estimated proportion of alveolar septa.

venous proportion (arterial BV5/LV r=0.41; $\mathrm{p}=0.09$ and venous BV5/LV $\mathrm{r}=0.54 ; \mathrm{p}=0.02$ ). Normalisation by NELV altered the correlations slightly (arterial: $r=0.47 ; \mathrm{p}=0.05$ and venous: $\mathrm{r}=0.10 ; \mathrm{p}=0.71$ ) (figure $5 \mathrm{~b}$ and $\mathrm{d}$ ). When combining the two arterial and venous measures, there was a direct correlation between increasing BV5/LV and percentage of alveolar septa $(r=0.54 ; \mathrm{p}=0.02)$; however, the correlation with \%hist-VCSA was no longer significant $(\mathrm{r}=0.18 ; \mathrm{p}=0.48)$.

When looking at specific arterial CT measures and evidence of arterial remodelling as measured by vessel morphometry, there was a negative correlation between arterial BV5/LV and \%Lumen Area $(r=-0.54$; $\mathrm{p}=0.02)$ and a positive correlation with \%Intima Area $(\mathrm{r}=0.48 ; \mathrm{p}=0.04)$. The relationship between arterial small vessel volume (arterial BV5/LV) with \%hist-VCSA was further explored with a linear regression model, adjusted for the CT-based measure of emphysema (\%LAA-950). In this model, increased distal arterial volume (arterial BV5/LV) remained a positive predictor of histological vascular cross-sectional area (\%hist-VCSA) even when adjusted for estimated emphysema obtained from the same CT scan (effect estimate \pm SE $0.0005 \pm 0.0001 ; \mathrm{p}=0.001 ;$ model $\mathrm{r}^{2}=0.51$ ).

\section{Discussion}

The goal of this study was to examine the relationship between CT-based markers of small vessel loss ("pruning") and the histological evidence of such vessel loss. In subjects with CT scans that allowed 3D vascular reconstruction (3D cohort) we accomplished this by using CT-derived measures of small vessel volume normalised either by lung volume or by the nonvascular NELV (arterial and venous BV5/LV and
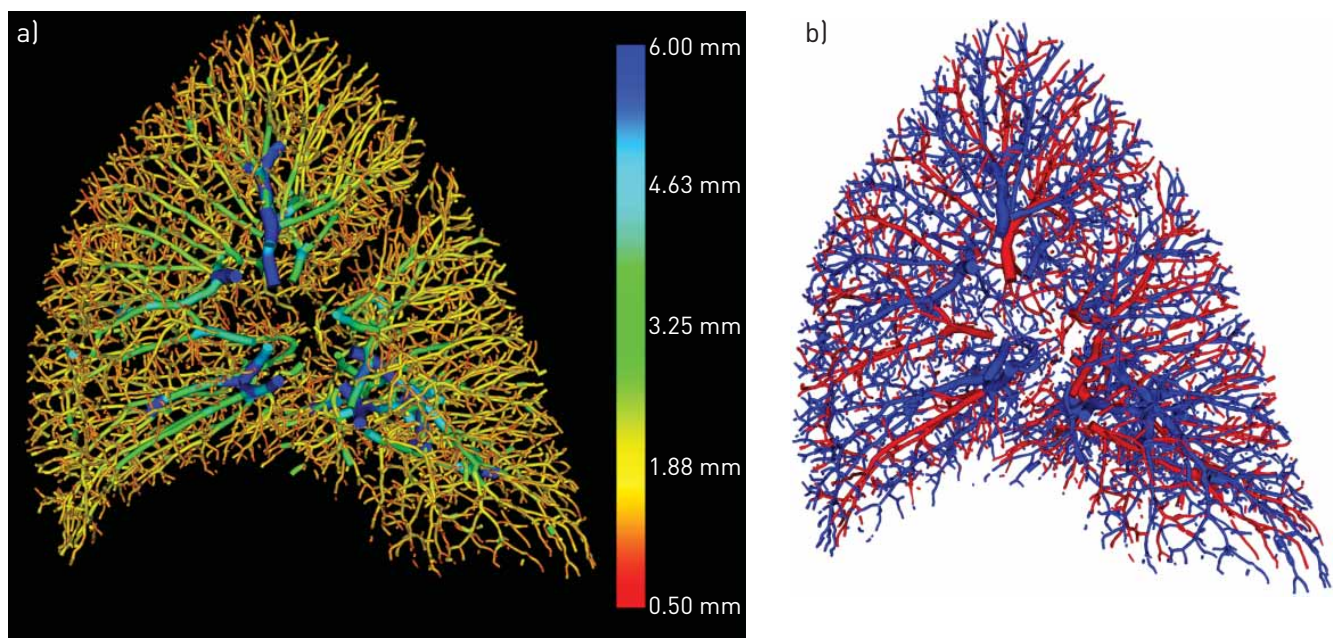

FIGURE 4 a) Example of three-dimensional reconstruction of a right lung where blue indicates larger vessels (radius $6 \mathrm{~mm}$ and cross-sectional area $\sim 110 \mathrm{~mm}^{2}$ ) and red indicates smaller vessels (radius $0.5 \mathrm{~mm}$ and cross-sectional area $0.8 \mathrm{~mm}^{2}$ ). b) Subsequent automated arterial and venous segmentation where blue denotes arteries and red denotes veins. 

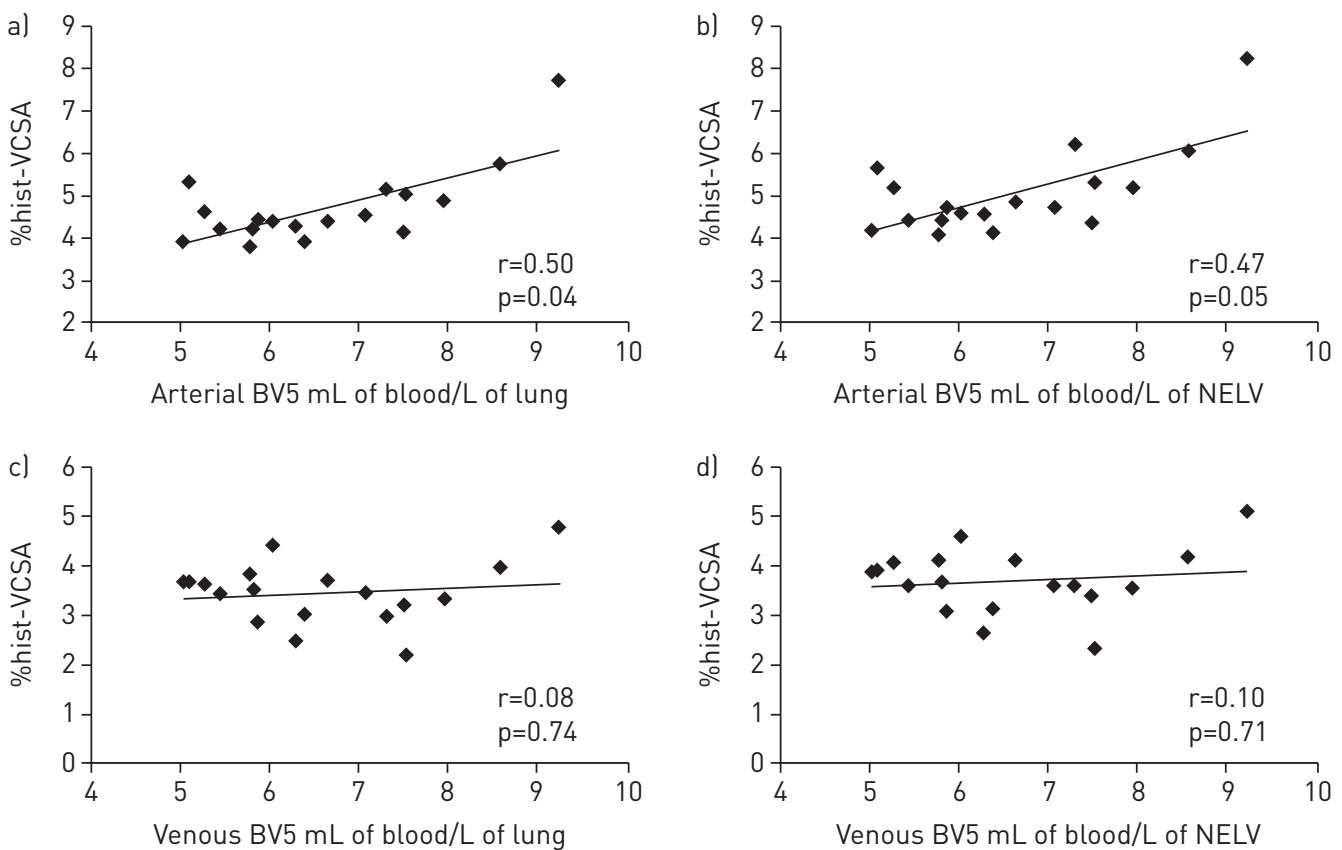

FIGURE 5 Relationship between estimated vascular volume in a, b) arterial and c, d) venous small vessels (blood vessel volume in vessels $<5 \mathrm{~mm}^{2}$ in cross-sectional area (BV5)) normalised by a, c) lung volume or b, d) tissue volume and histological metrics of small vessel (diameter $<500 \mu \mathrm{m}$ ) density (histological vessel cross-sectional area (\%hist-VCSA)) and the estimated proportion of alveolar septa. Correlation coefficients are nonparametric (Spearman). NELV: nonemphysematous lung volume.

BV5/NELV, respectively). In a separate series of subjects with thin enough sections and uniform image type (but not of sufficiently high quality for $3 \mathrm{D}$ reconstruction), we performed a $2 \mathrm{D}$ equivalent of this method where the vascular cross-sectional area was divided by the lung slice area (CSA5\%). A histological equivalent to these measures was proposed and measured, in which the cross-sectional area of vessels $<500 \mu \mathrm{m}$ of diameter was divided by the total area of the tissue being examined (\%hist-VCSA), as well as the estimated proportion of alveolar septa in lung tissue. In both analyses, imaging-based markers of small vessel volume loss correlated with their histological counterparts and were also related to measures of alveolar destruction (presence/absence of alveolar septa).

While tissue destruction due to emphysema is one mechanism of vascular impairment in smokers, multiple other pathways including those modulated by inflammatory mediators, vessel remodelling, hypoxic vasoconstriction and compression due to air trapping have been described as leading to pulmonary vascular disease. In addition, multiple cardiovascular comorbidities exist that confound smoking exposure and may also affect the pulmonary vasculature (e.g. as is the case in left-sided heart failure). In our study we observed these comorbidities in six subjects (31.5\%) in the 2D cohort and 12 subjects $(67 \%)$ in the $3 \mathrm{D}$ cohort. The degree to which each of these mechanisms affects the microvasculature (apparent on histology) and macrovasculature (apparent on CT) is unknown.

This study shows that there is a relationship between changes of vascular density as assessed by histology and as measured by CT scans. This relationship may be partially mediated by alveolar destruction as evidenced by correlations with the proportion of alveolar septa. The \%LAA-950 when added to a predictive model of the tissue vascular density did not eliminate the predictive power of arterial BV5/LV. It should be noted that in this cohort of patient candidates for lung resection with normal or only mildly impaired pulmonary function, the extent of emphysema visible on CT scan as assessed by \%LAA-950 was small (table 3), suggesting that microscopic alveolar destruction might be present before CT evidence of emphysema is apparent. Examining the relationship between vascular changes in histology and those noted on imaging may improve our understanding of the role of the vasculature in the progression of lung disease in smokers.

Interestingly, measurements of arterial small vessel density (arterial BV5/LV) did correlate with morphometric assessments of vessel remodelling in pulmonary muscular arteries. Increasing arterial small vessel density was associated with a narrower arterial lumen and thicker intima. One potential explanation for this correlation is that in the context of the loss of vascular density as measured by CT, the 
microvasculature that is not lost has a different vascular morphology as a subgroup. Another explanation is that the reduction of small vessel density assessed by CT likely reflects vascular pruning resulting from lung tissue destruction rather than from narrowing of small pulmonary arteries and that these are separate phenomena that might eventually concur in producing pulmonary hypertension in advanced disease stages.

This study demonstrates that CT-based imaging findings of loss of distal vasculature are accompanied by loss of vascular cross-sectional area on histology. Vascular remodelling has been described as an important component of the development of pulmonary vascular disease [7], including pulmonary arterial hypertension $[28,29]$. Loss of distal vasculature and changes to the vascular structure assessed using CT imaging have been described in asthma [30], smoking-related pulmonary vascular and cardiovascular disease $[14,18,19]$, pulmonary arterial hypertension $[31,32]$, and chronic thromboembolic pulmonary hypertension [33]. These changes include proximal arterial dilation, distal loss of vasculature and increased vascular tortuosity as well as overall vascular tree complexity. Understanding the relationship between imaging measurements and histological measurements will enhance the utility of imaging techniques for quantification of vascular remodelling both in disease progression as well as in response to therapy [34].

One limitation of the present study is that our cohort consists of subjects with a diagnosis of lung cancer that may have altered the underlying anatomical and physiological parameters, although the histological assessments were performed in areas far from the neoplasm and without apparent tissue distortion. Since lungs were not fixed at a constant inflation, some areas of lung tissue could be collapsed and produce alterations in the measurements of morphometry, but to minimise this possibility tissue was distended in PBS prior to fixation. To minimise the effect of this on vascular morphometry, a theoretical diameter of the fully distended artery was calculated by dividing the length of the external elastic lamina by $\pi$ as described in a previous study of vascular morphometry [35]. Subjects were selected retrospectively, which may have introduced some selection bias into the study. While cohort selections were made in part to minimise heterogeneity in CT acquisition, some variability in image acquisition existed within each cohort. Most patients with chronic obstructive pulmonary disease (COPD) used in this study had Global Initiative for Chronic Obstructive Lung Disease stage I or II disease and thus significant COPD burden is not well represented in this dataset. Similarly, the burden of emphysema, as assessed by CT scan, was low, and thus there were not significant differences between calculated lung volume and lung volume adjusted for emphysema. In prior studies the ratio of the small vessel volume to total blood volume has also been proposed as an index of vascular remodelling. This index, however, represents a fundamentally different type of fractional measurement, which while similarly unit-less, can be affected both by loss of distal volume as well as increase in proximal volume, and thus is difficult to compare with the histological measure of vascular density. The vascular density by histology includes veins and arteries because of the difficulty of differentiating them due to vessel size $(<500 \mu \mathrm{m}$ diameter $)$ and sample distortion.

In summary, results of the present study demonstrate that CT assessments of small vessel density reflect the number of small pulmonary vessels, which in smokers and patients with COPD is closely related to the integrity of lung parenchyma. This provides evidence for the utility of imaging-based methods of vascular quantification on a macro scale as a marker of vascular pruning on a histological scale.

Conflict of interest: F.N. Rahaghi has nothing to disclose. G. Argemi has nothing to disclose. P. Nardelli has nothing to disclose. D. Dominguez-Fandos has nothing to disclose. P. Arguis has nothing to disclose. V.I. Peinado has nothing to disclose. J.C. Ross reports grants from NIH, during the conduct of the study. S.Y. Ash has nothing to disclose. I. de La Bruere has nothing to disclose. C.E. Come reports grants from NIH/NHLBI (K23HL114735), during the conduct of the study. A.A. Diaz has nothing to disclose. M. Sanchez has nothing to disclose. G.R. Washko reports grants from NIH and BTG Interventional Medicine, grants from and has provided consultancy and participated on advisory boards for Boehringer Ingelheim, has provided consultancy for Genentech, Regeneron and GlaxoSmithKline, has provided consultancy and participated on data and safety monitoring boards for PulmonX, participated on advisory boards for ModoSpira and Toshiba, grants from and has provided consultancy for Janssen Pharmaceuticals, outside the submitted work; and is a founder and co-owner of Quantitative Imaging Solutions, which is a company that provides image-based consulting and develops software to enable data sharing; in addition, G.R. Washko's spouse works for Biogen, which is focused on developing therapies for fibrotic lung disease. J.A. Barberà has nothing to disclose. R. San Jose Estepar reports grants from NHLBI, personal fees from Toshiba and Boehringer Ingelheim, outside the submitted work; and is also a founder and co-owner of Quantitative Imaging Solutions, which is a company that provides image-based consulting and develops software to enable data sharing.

Support statement: Authors in this study were supported by NHLBI grants 5T32HL007633, K23HL136905 (F.N Rahaghi), 1R01HL116931 and 1R01HL116473 (R. San Jose Estepar and G.R. Washko); and grants PS0900536 from Institute of Health Carlos III (ISCIII) (J.A. Barberà) and 2017SGR617 from Agència de Gestió d'Ajuts Universitaris i de Recerca (J.A. Barberà), and an educational grant from Menarini, Spain. Funding information for this article has been deposited with the Crossref Funder Registry. 


\section{References}

1 Liebow AA. Pulmonary emphysema with special reference to vascular changes. Am Rev Respir Dis 1959; 80: 67-93.

2 Reid JA, Heard BE. The capillary network of normal and emphysematous human lungs studied by injections of Indian ink. Thorax 1963; 18: 201-212.

3 Kessler R, Faller M, Weitzenblum E, et al. "Natural history" of pulmonary hypertension in a series of 131 patients with chronic obstructive lung disease. Am J Respir Crit Care Med 2001; 164: 219-224.

4 Chaouat A, Naeije R, Weitzenblum E. Pulmonary hypertension in COPD. Eur Respir J 2008; 32: $1371-1385$.

5 Borden CW, Wilson RH, Ebert RV, et al. Pulmonary hypertension in chronic pulmonary emphysema. Am J Med 1950; 8: 701-709.

6 Barr RG. The epidemiology of vascular dysfunction relating to chronic obstructive pulmonary disease and emphysema. Proc Am Thorac Soc 2011; 8: 522-527.

7 Barbera JA. Mechanisms of development of chronic obstructive pulmonary disease-associated pulmonary hypertension. Pulm Circ 2013; 3: 160-164.

8 Peinado VI, Pizarro S, Barbera JA. Pulmonary vascular involvement in COPD. Chest 2008; 134: 808-814.

9 Voelkel NF, Gomez-Arroyo J, Mizuno S. COPD/emphysema: the vascular story. Pulm Circ 2011; 1: 320-326.

10 Blanco I, Piccari L, Barbera JA. Pulmonary vasculature in COPD: the silent component. Respirology 2016; 21: 984-994.

11 Matsuoka S, Washko GR, Dransfield MT, et al. Quantitative CT measurement of cross-sectional area of small pulmonary vessel in COPD: correlations with emphysema and airflow limitation. Acad Radiol 2010; 17 : 93-99.

12 Estepar RS, Kinney GL, Black-Shinn JL, et al. Computed tomographic measures of pulmonary vascular morphology in smokers and their clinical implications. Am J Respir Crit Care Med 2013; 188: 231-239.

13 Hale KA, Niewoehner DE, Cosio MG. Morphologic changes in the muscular pulmonary arteries: relationship to cigarette smoking, airway disease, and emphysema. Am Rev Respir Dis 1980; 122: 273-278.

14 Matsuoka S, Washko GR, Yamashiro T, et al. Pulmonary hypertension and computed tomography measurement of small pulmonary vessels in severe emphysema. Am J Respir Crit Care Med 2010; 181: 218-225.

15 Santos S, Peinado VI, Ramirez J, et al. Characterization of pulmonary vascular remodelling in smokers and patients with mild COPD. Eur Respir J 2002; 19: 632-638.

16 Santos S, Peinado VI, Ramirez J, et al. Enhanced expression of vascular endothelial growth factor in pulmonary arteries of smokers and patients with moderate chronic obstructive pulmonary disease. Am J Respir Crit Care Med 2003; 167: 1250-1256.

17 Vlahovic G, Russell ML, Mercer RR, et al. Cellular and connective tissue changes in alveolar septal walls in emphysema. Am J Respir Crit Care Med 1999; 160: 2086-2092.

18 Rahaghi FN, Wells JM, Come CE, et al. Arterial and venous pulmonary vascular morphology and their relationship to findings in cardiac magnetic resonance imaging in smokers. J Comput Assist Tomogr 2016; 40: 948-952.

19 Washko GR, Nardelli P, Ash SY, et al. Arterial vascular pruning right ventricular size and clinical outcomes in COPD. Am J Respir Crit Care Med 2019; in press [https://doi.org/10.1164/rccm.201811-2063OC].

20 Schneider CA, Rasband WS, Eliceiri KW. NIH Image to ImageJ: 25 years of image analysis. Nat Methods 2012; 9: 671-675.

21 Ross JC, Estepar RS, Diaz A, et al. Lung extraction, lobe segmentation and hierarchical region assessment for quantitative analysis on high resolution computed tomography images. Med Image Comput Comput Assist Interv 2009; 12: 690-698.

22 Estepar RS, Ross JC, Krissian K, et al. Computational vascular morphometry for the assessment of pulmonary vascular disease based on scale-space particles. Proc IEEE Int Symp Biomed Imaging 2012: 1479-1482.

23 Kindlmann GL, San Jose Estepar R, Smith SM, et al. Sampling and visualizing creases with scale-space particles. IEEE Trans Vis Comput Graph 2009; 15: 1415-1424.

24 Nardelli PLM, Møller CB, Andersen A-SH, et al. Accurate measurement of airway morphology on chest CT images. Lect Notes Comput Sci 2018; 11040: 335-347.

25 Matsuoka S, Yamashiro T, Washko GR, et al. Quantitative CT assessment of chronic obstructive pulmonary disease. Radiographics 2010; 30: 55-66.

26 Madani A, Zanen J, de Maertelaer V, et al. Pulmonary emphysema: objective quantification at multi-detector row CT - comparison with macroscopic and microscopic morphometry. Radiology 2006; 238: 1036-1043.

27 Nardelli P, Jimenez-Carretero D, Bermejo-Pelaez D, et al. Pulmonary artery-vein classification in CT images using deep learning. IEEE Trans Med Imaging 2018; 37: 2428-2440.

28 Chaudhary KR, Taha M, Cadete VJ, et al. Proliferative versus degenerative paradigms in pulmonary arterial hypertension: have we put the cart before the horse? Circ Res 2017; 120: 1237-1239.

29 Vonk-Noordegraaf A, Marcus JT, Holverda S, et al. Early changes of cardiac structure and function in COPD patients with mild hypoxemia. Chest 2005; 127: 1898-1903.

30 Ash SY, Rahaghi FN, Come CE, et al. Pruning of the pulmonary vasculature in asthma. The Severe Asthma Research Program (SARP) Cohort. Am J Respir Crit Care Med 2018; 198: 39-50.

31 Helmberger M, Pienn M, Urschler M, et al. Quantification of tortuosity and fractal dimension of the lung vessels in pulmonary hypertension patients. PLoS One 2014; 9: e87515.

32 Moledina S, de Bruyn A, Schievano S, et al. Fractal branching quantifies vascular changes and predicts survival in pulmonary hypertension: a proof of principle study. Heart 2011; 97: 1245-1249.

33 Rahaghi FN, Ross JC, Agarwal M, et al. Pulmonary vascular morphology as an imaging biomarker in chronic thromboembolic pulmonary hypertension. Pulm Circ 2016; 6: 70-81.

34 Rahaghi FN, Winkler T, Kohli P, et al. Quantification of the pulmonary vascular response to inhaled nitric oxide using noncontrast computed tomography imaging. Circ Cardiovasc Imaging 2019; 12: e008338.

35 Barbera JA, Riverola A, Roca J, et al. Pulmonary vascular abnormalities and ventilation-perfusion relationships in mild chronic obstructive pulmonary disease. Am J Respir Crit Care Med 1994; 149: 423-429. 